\title{
JLCORS Observation Data Quality Checking and Assessment
}

\author{
Junqing Liu ${ }^{1,2}$ \\ 1. College of Geo-Exploration Science and Technology \\ Jilin University \\ 2. Earthquake Administration of JiLin Province \\ Changchun, China \\ e-mail: woxin5295@yahoo.com \\ Cai Liu* \\ College of Geo-Exploration Science and Technology \\ Jilin University \\ Changchun, China \\ e-mail:liucai@jlu.edu.cn \\ * Corresponding Author
}

\author{
Guodong Yang \\ College of Geo-Exploration Science and Technology \\ Jilin University \\ Changchun, China \\ e-mail: ygd@liu.edu.cn
}

\author{
You Tian \\ College of Geo-Exploration Science and Technology \\ Jilin University \\ Changchun, China \\ e-mail:tianyou@jlu.edu.cn
}

\begin{abstract}
To adopt teqc software to carry out comprehensive checking and assessment on 49 continuous running observation stations from the JLCORS network system, including data integrity, ionospheric delay and variability, multipath effect etc. Besides, data sampling rate adopts 30 seconds and the satellite cut-off angle is 10 degrees. The result reveals that $95 \%$ observation station sampling efficiency is between 0.9 and 1 and $80 \%$ CSR value is between 0.0 and 0.35 ; the RMS of the MP1, MP2 is between 0.15 and $0.45 \mathrm{~m}$ and the maximum value of MP1 and MP2 is $0.45 \mathrm{~m}$ of SLAN station and $0.42 \mathrm{~m}$ of DEFN station. The operating condition of receiving set is good and detection environment as well as data are in accordance with the requirements of Technical specification for Crustal Movement Observation Network of China and are better than the standard of international IGS station. In addition, we carry out statistical analysis on the time interval with serious delays in terms of ionosphere as well as unhealthy satellite so as to provide reliable foundation for GNSS postprocessing.
\end{abstract}

Keywords-GNSS;CORS;TEQC;Multipath effect;Earth movement

\section{INTRODUCTION}

GNSS continuously operating reference system (CORS for short) provides a dazzling array of high-accurate, dynamic and all-day convenient services in terms of real time positioning, navigation and time service. What is worth paying attention to is that its emergence can be beckoned as a milestone for spatial measurement modern development. At present, it is widely used in land surveying and mapping, urban construction and planning, land administration, deformation monitoring of building, traffic monitoring, environment monitoring, earthquake prevention etc. A dazzling array of countries has established their own CORS network. For example, in
America, NGS is responsible to establish 160 CORS network composed by more than 160 stations; in England, the CPGRS network is a CPRS network composed by more than 60 GNSS stations; and in Germany, Japan,and India, there are large scale CORS network. In China, the first CORS network is established in Shenzhen and there are CORS systems with comprehensive functions in Jiangsu ${ }^{[1]}$, Sichuan and Shanghai.

Different applications have different requirement on the accuracy of GNSS and general navigation time does not need a high level of accuracy yet researches on deformation monitoring or plate motion requires submillimeter accuracy ${ }^{[2,3]}$. What's more, site selection, environment observation and data quality are highly demanded because it is necessary to test the CORS indicators as well as the comprehensive quality ${ }^{[4]}$. This paper carries out a full test and evaluation on the observation quality based on calculating various indicators of all stations of JLCORS.

\section{INTRODUCTION OF JILIN CONTINUOUSLY OPERATING REFERENCE SYSTEM}

Jilin Continuously Operating Reference System JLCORS is jointly established by the mapping Bureau of Jilin province,Jilin Meteorological Bureau Jilin Seismological Bureau with 57 stations and the average distance is $60 \mathrm{~km}$ which can cover $90 \%$ areas of Jilin.It is a provincial level GNSS system which establishes an eternal references system via internet to construct a new earth measurement system. Besides, it comprehensively adopts the GNSS technology in terms of earth measurement, engineering measurement, meteorological monitoring, ground settlement monitoring and city geographic information system which organically integrates satellite navigation positioning technology, surveying and mapping, meteorology, and modern communication technology etc. In December 2012, 49 stations were put into use and the station distribution can be seen in figure 1 . The controlling 
center of CORS acquires GNSS data and carries out unified calculation to get the correction value of systematic errors in the network so as to establish a error correcting model covering the whole network and send the correct values to users. While users receiving these correct data, they are able to obtain a high precision positioning result in real time of after ${ }^{[5]}$.

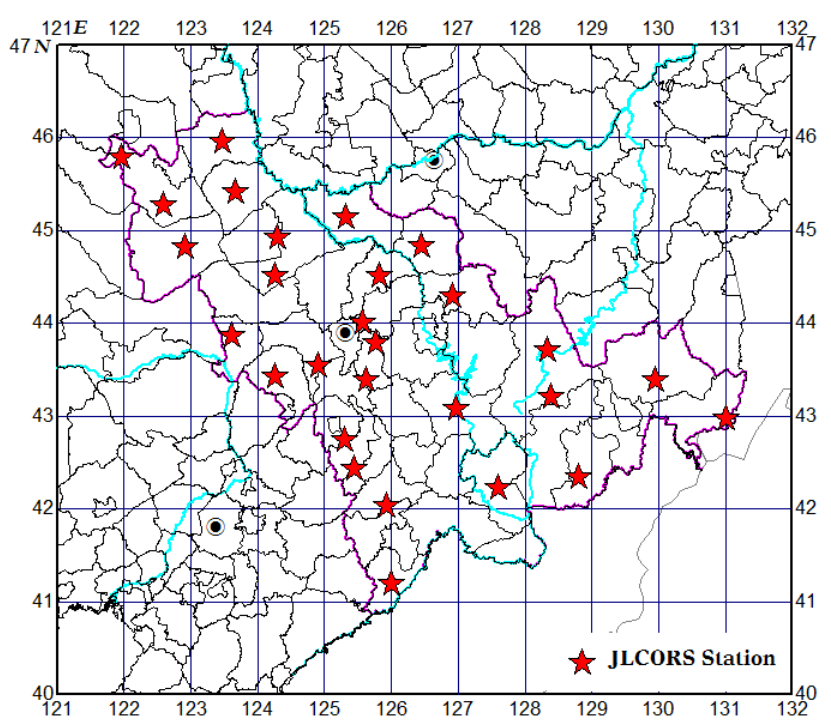

Figure 1. JLCORS reference station

\section{TEQC SOFTWARE INTRODUCTION}

Teqc (Translation, Editing and Quality Checking) is a powerful preprocessing software for GNSS, which is developed by UNAVCO and completed by C language, which can be adopted in dos and linux. Its main functions include standardization of observation data for various GNSS receivers (binary to the RINEX format), data editing and data quality assessment. In terms of earth movement, and deformation monitoring GNSS requires sub-millimeter accuracy, which ask higher requirement in terms of station establishment and observation environment selecting. Observation quality evaluation includes the proportion of comprehensive observation data to total observation, total epoch to cycle slips, the signalnoise ratio of receivers, multipath of L1 and L2, among which the calculation formula for multipath shows in the following:

$$
\begin{aligned}
& M P_{1}=\rho_{1}-\left(\frac{\alpha+1}{\alpha-1}\right) \varphi_{1}+\left(\frac{2}{\alpha-1}\right) \varphi_{2} \\
& M P_{2}=\rho_{2}-\left(\frac{2 \alpha}{\alpha-1}\right) \varphi_{1}+\left(\frac{\alpha+1}{\alpha-1}\right) \varphi_{2}
\end{aligned}
$$

In the formula, $\rho_{1}$ and $\rho_{2}$ are Pseudo-range observation data for L1 and L2 while $\varphi_{1}$ and $\varphi_{2}$ are phase observation data and $\alpha$ is the frequency ratio which is a constant.

\section{PrePare Your PAPER Before Styling}

It adopts 24-hour continuous observation data on 1st January 2014, and all data sample rate is 30 seconds. What's more, it adopts teqc to carry out various indicator calculation, including supposing data (\#expt), real data (\#have) and data utilization ratio, L1 multipath effect (MP1), L2 multipath effect (MP2), observation data and cycle slip ratio (o/slps), $\operatorname{IOD}\left(10^{\circ}\right)$ and parts of the station results show in table 1.

\section{A. Analysis on the data integrity}

Sampling efficiency refers to the ratio of maximum collecting epoch to theoretical epoch during a certain period which can be beckoned as the comprehensive reflection of the surrounding station environment ${ }^{[6,7]}$. CSR is the ratio of cycle slip to the maximum collecting epoch and in reality, it should be multiplied by 1000 for readability and the formula is shown as follows:

$$
C S R=1000 \times \frac{s l p s}{o}
$$

In the formula, slps refers to cycle slip and o refers to the maximum collecting epoch. The rest result of JLCORS (tab.1 and fig.2) reveals that the sampling efficiency of 95\% observation station is between 0.9 and $1,0.95$ and 1.0 for 33 stations, the comprehensive observation environment is good. The CSRT value of $80 \%$ stations is between 0.00 and 0.35 while the maximum is 1.66 of BAIC station, the CSR standard of international IGS is less than 10. Therefore, we can see that the data integrity of JLCORS is in accordance with the basic requirement and the overall indicator is superior to international IGS station.
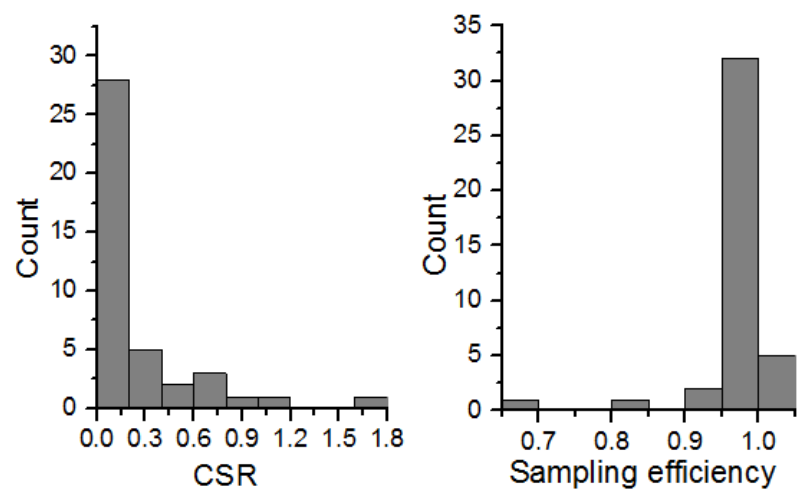

Figure 2. CSR and sampling efficiency histogram from JLCORS reference stations 
TABLE 1 TEQC TESTING RESULT

\begin{tabular}{|c|c|c|c|c|c|c|c|}
\hline $\begin{array}{l}\text { Station } \\
\text { name }\end{array}$ & $\begin{array}{l}\text { Observation } \\
\text { length (hrs) }\end{array}$ & $\begin{array}{c}\text { Supposing } \\
\text { collecting } \\
\text { Data (\#expt) }\end{array}$ & $\begin{array}{c}\text { Real } \\
\text { collecting } \\
\text { data } \\
\text { (\#have) }\end{array}$ & $\begin{array}{c}\text { Data } \\
\text { efficiency } \\
(\%)\end{array}$ & MP1 & MP2 & $0 /$ slps \\
\hline ANTU & 24 & 24976 & 24971 & 100 & 0.26 & 0.28 & 24971 \\
\hline BAIC & 24 & 24883 & 24669 & 99 & 0.28 & 0.29 & 602 \\
\hline BAIS & 24 & 24958 & 24576 & 98 & 0.33 & 0.34 & 4915 \\
\hline CBAI & 24 & 25000 & 24393 & 98 & 0.34 & 0.36 & 24393 \\
\hline CHAC & 24 & 24893 & 24816 & 100 & 0.25 & 0.23 & 1460 \\
\hline CHYA & 24 & 24895 & 24895 & 100 & 0.15 & 0.18 & 24895 \\
\hline DAAN & 24 & 24818 & 24818 & 100 & 0.30 & 0.36 & 24818 \\
\hline DEFN & 24 & 24929 & 24929 & 100 & 0.26 & 0.42 & 24929 \\
\hline$\overline{\text { DGAN }}$ & 24 & 24985 & 24982 & 100 & 0.32 & 0.33 & 24982 \\
\hline DHUA & 24 & 24960 & 24945 & 100 & 0.26 & 0.28 & 12473 \\
\hline DHUI & 24 & 24960 & 24945 & 100 & 0.26 & 0.28 & 12473 \\
\hline EDAO & 24 & 24987 & 24718 & 99 & 0.34 & 0.40 & 3090 \\
\hline FJTN & 24 & 24892 & 24755 & 99 & 0.21 & 0.19 & 917 \\
\hline FUYU & 24 & 24882 & 24845 & 100 & 0.17 & 0.17 & 24845 \\
\hline GJZI & 24 & 24862 & 24846 & 100 & 0.28 & 0.31 & 24862 \\
\hline GZLN & 24 & 24886 & 24860 & 100 & 0.35 & 0.39 & 24886 \\
\hline HCUN & 24 & 15279 & 12668 & 83 & 0.37 & 0.25 & 12668 \\
\hline HDAN & 24 & 24951 & 24925 & 100 & 0.38 & 0.37 & 6231 \\
\hline JIAN & 24 & 24961 & 24699 & 99 & 0.15 & 0.19 & 6175 \\
\hline JIUT & 24 & 24904 & 24875 & 100 & 0.21 & 0.26 & 12438 \\
\hline
\end{tabular}

\section{B. Analysis on the multipath}

Multipath is an important source of error in GNSS testing and its emergence is caused by the GNSS signal reflection of surrounding architectures, water surface or other smooth surfaces. Besides, the unwanted signal of the receiver is weak yet greatly affected the calculation of pseudo-range and the positioning error can reach $30 \mathrm{~m}^{[6,8]}$. In order to avoid or weaken the path effect, the location of GNSS station should be away from large reservoirs, buildings, and mountains so as to avoid the condition that the reflection shall affect the signals. However, because of limitations of station construction conditions, we cannot always have ideal locations which requires us to carry out multipath efficiency. After data-processing, we can add the receiving cut-off angle or shield the satellite during a certain period so as to improve the positioning accuracy.
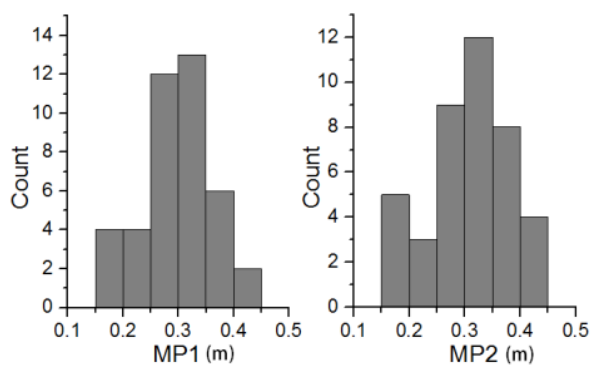

Figure 3. MP1 and Mp2 histogram from JLCORS reference stations
IGS has once calculated the multipath for the fixed stations and the average MP1 of 2/3 observation stations is less than than $0.5 \mathrm{~m}$, and the average MP2 is less than $0.75 \mathrm{~m}$. We design the multipath based on this result, namely, MP1 $<=0.5 \mathrm{~m}, \mathrm{MP} 2<=0.5 \mathrm{~m}$. According to Chinese standard, MP1< $=0.5 \mathrm{~m}, \mathrm{MP} 2<=0.5 \mathrm{~m}$ means that the smaller the data the poorer effect of the multipath. The MP2 multipath of JLCORS result (table 1 and figure 3) shows that two indicators of all stations are between 0.15 and 0.45 and the value of $80 \%$ stations is between 0.25 and $0.35 \mathrm{~m}$, and the maximum value of MP1 and MP2 is $0.45 \mathrm{~m}$ of SLAN and $0.42 \mathrm{~m}$ of DEFN. All of the multipath efficiency should be in accordance with the requirement of Technical Specification for Crustal Movement Observation Network of China and be superior to global IGS station.

\section{Analysis on the Ionospheric delay and change rate}

The ionospheric delay is the main error source of GNSS observation which cannot be avoided so we have to combine the L1 and L2 so as to eliminate the errors. The signal transmission path of ionospheric delay can be shown in the following integral formula:

$$
d_{i o n}=-C \frac{40.28}{f^{2}} \int_{s^{\prime}} N_{e} d s
$$

In the formula, $N_{e}$ refers to ionospheric intensity, and the unit is electronic number $/ \mathrm{m}^{3}$; $f$ refers to signal frequency and the unit is $\mathrm{Hz}$; $\mathcal{C}$ refers to the speed of light 
in vacuum and the unit is $\mathrm{m} / \mathrm{s} ; s^{\prime}$ refers to the GNSS signal transmission path.

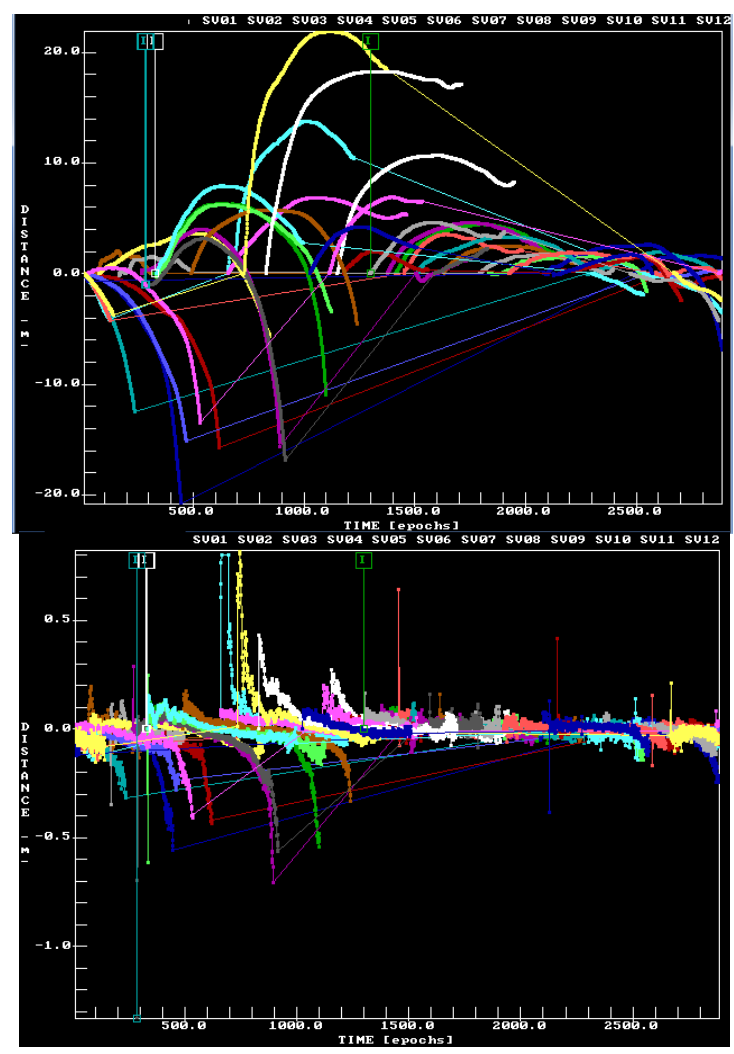

Figure 4. Ionospheric delay and its change rate in ANTU station

Teqc adopts the comparison expression to have the ionospheric delay and change rate and the output document is *.ion and *.iod. This paper deals with 49 stations and acquires observation materials during the period with ionospheric delay and drastic change which can provide references for selecting time and healthy satellite of base line post-processing ${ }^{[9]}$. The part error will bring $30 \mathrm{~m}$ to $100 \mathrm{~m}$ error so it is a key sector for choosing proper observation time and getting rid of unhealthy satellite so as to have accurate GNSS calculation ${ }^{[10]}$. Fig. 4 shows the ionospheric delay and change rate curve of 2880 epochs of ANTU station. In the figure, between epoch 700 and 2000, the ionospheric delay of four satellite reach $20 \mathrm{~m}$. After observation, we can know that the four satellites are SV04, SV09, SV16 and SV 26. Therefore, in postprocessing, we have to shield the observation data of these four satellites.

\section{CONCLUSIONS}

By carrying out comprehensive observation on various indicators of JCCORS, we hold the idea that the observation environment is in accordance with the requirement of GNSS and various indicators are in accordance with the standards of Technical Specification for Crustal Movement Observation Network of China which are obviously superior to international IGS station. Concerning the error source of positioning accuracy, the multipath efficiency, the JLCORS station effect is poorer than IGS standard. Concerning some large observation stations, we can improve the environment or add the antidiameter so as to weaken the effect. To conclude, the receivers of various observation stations operate well with relatively good observation quality.

\section{ACKNOWLEDGMENT}

The research work supported by Science for Earthquake Resilience (No. XH14016Y) from China Earthquake Administration.

\section{REFERENCES}

[1] Zhao Qian, Shen Fei. Research on the IGS station selection of Jiangsu CORS calculaiton[J].Science of serving and mapping, 2011, (06): 124-5+76.

[2] Liu Junqing, Li Ke, Liang Guojing et al. GPS data quality detection and analysis of Tianchi volcano, Changbaishan [J]. Seismological and Geomagnetic Observation and Research, 2010, (02): 83-7.

[3] Liu Junqing, Ding Guang, Zhang Chenxia et al. GPS receiver noise and Antenna Phase Center Bias test technology application[J]. Seismological Research of Northeast China, 2012, (03): 77-81.

[4] Li Jun, Wang Jiye, Xiong Xiong et al. GPS data quality detection and analysis of Northeast Asia[J]. Wuhan University Journal of Natural Sciences, 2006, (03): 209-12.

[5] Feng Yong, Guo Yi. The CORS principle and application of GPS[J]. JOURNAL OF INNER MONGOLIA UNIVERSITY OF SCIENCE AND TECHNOLOGY, 2010, (04): 298-301.

[6] Tang Liming, Li Chenggang, Zhang Jianguo et al. Research on the dynamic displacement of ground surface observation technology in Precision area of GPS/CORS[J]. Surveying And Mapping Bulletin, 2010, (05): 6-9.

[7] Bo Liu, Xiao Ruan, Hu Youjian. Research on the acuracy and stability of CORS [J]. Journal of henan polytechnic university natural science, 2005, (04): 283-8.

[8] Jiang Weiping, Yuan Peng, Tian Zhi et al. The unified approach for criterion datum of CORS[J] Wuhan University Journal of Natural Sciences, 2014, (05): 566-70.

[9] Li Xiaoliang. The construction and application of CPRS[D]; China University of Geosciences, 2014.

[10] Geng Changjiang, Tang Weiming, Guo Hongping. Monitoring the ionospheric change with CORS $[\mathrm{J}]$. Journal of Geodesy and Geodynamics, 2008,(05):105-108. 